\title{
A DETERMINATION OF PULSAR EMISSION GEOMETRY FROM POLARIZATION OBSERVATIONS
}

\author{
XINJI WU \\ CCAST (World Laboratory) and Department of Geophysics, Peking University \\ WEN XU \\ Department of Physics, Peking University
}

\begin{abstract}
One of the important problems in pulsar studies is to determine the magnetic inclination angle $\alpha$, the intrinsic width of the radiation beam $(2 \rho)$ and the angle $(\alpha+\beta)$ between the observer's direction and the rotation axis. In this paper we solve this problem for individual pulses by using the observed pulse width $(2 \Delta \phi)$, the swing of polarization angle $(2 \Delta \psi)$, and its central gradient $(d \psi / d \phi)_{\max }$.

From the polar cap model we establish three basic geometrical relations, a complete set of equations from which explicit solutions can be obtained using the observed data. This is the first time that the orientation of pulsar emission is solved analytically solely on the basis of a geometrical model. However, the results are shown to be sensitively connected to the polarization-angle swing $(2 \Delta \psi)$, which is not well measured for most pulsars. So the number of pulsars to which our method can be applied is limited. The importance of the measurement of $\Delta \psi$ is seen from our method. To ensure the credibility of our results, we have discussed the conditions to be satisfied by all reliable pulsar measurements. Our method is shown to be more favorable for pulsars with large pulse width $2 \Delta \phi$, large central gradient $(d \psi / d \phi)_{\max }$ and large magnetic inclination angle $\alpha$. Out of 120 pulsars (from Lyne and Manchester 1988), 40 are solvable, and 7 are believed to be reliable. We discuss our method for the determination of pulsar geometry in comparison with other methods.
\end{abstract}

\section{Introduction}

One of the important problems in pulsar studies is to determine the magnetic inclination angle $\alpha$, the intrinsic width of the radiation beam $(2 \rho)$, and the angle $(\alpha+\beta)$ between the observer's direction and the rotation axis. Early discussions were focused on the magnetic inclination $\alpha$, which is theoretically given by Jones (1977) as

$$
\tan ^{2} \alpha=\tau_{D} \frac{\dot{P}}{P}
$$

Investigations of the distribution of $\alpha$ based on the statistics of apparent beamwidth by Prószyński et al., (1979) and Wu et al., (1982) showed that the magnetic inclination tends to be small. But their method does not work for every pulsar. A significant improvement was made by Narayan and Vivekanand (1982) who made a least-squares fit to the observed $\psi-\phi$ curve of polarization-angle variation. They estimated $\alpha$ and $\beta$ for seven pulsars. They also described a method in which the observed polarization-angle gradients at both the main pulse and interpulse are used to estimate $\alpha$ and $\beta$

$$
\left(\frac{d \psi}{d \phi}\right)_{\max }=\frac{\sin \alpha}{\sin \beta}
$$

and

$$
\left(\frac{d \psi}{d \phi}\right)_{\text {interpulse }}=-\frac{\sin \alpha}{\sin (2 \alpha+\beta)} .
$$

Another well known geometrical relation is

$\cos \rho=\cos \Delta \phi \sin (\alpha+\beta) \sin \alpha+\cos (\alpha+\beta) \cos \alpha$

Using eqs.(1), (2) and (4), the statistical result given by Wu et al. (1985) shows

$$
\rho=7.1^{\circ} P^{-0.67} \text {. }
$$

Guseinov and Yusifov gave ranges of $\alpha$ and $\rho$ for pulsars in their article (1985).

$$
\rho=10^{\circ} P^{-0.7} \sim 4^{\circ} P^{-0.7} .
$$

The statistical result given by Lyne and Manchester (1988) shows

$$
\rho=6.5^{\circ} P^{-1 / 3} \text {. }
$$

Each method can give the values of $\alpha, \beta$ and $\rho$, but the values are different for each model. We hope to determine $\alpha, \beta$ and $\rho$ without such ambiguity from the observed data. This leads us look back on the polar cap model, especially the method of least-squares fit to the curve, and question whether we can make our determination solely on geometrical assumptions. Fortunately, we have the observed polarization position angle given by

$$
\tan \Delta \psi=\frac{\sin \alpha \sin \Delta \phi}{\sin (\alpha+\beta) \cos \alpha-\cos (\alpha+\beta) \sin \alpha \cos \Delta \phi}
$$


where $\psi$ is the position angle of the projected direction of the pulsar rotation axis, $2 \Delta \psi$ is the swing of the polarization position angle, $2 \Delta \phi$ is the apparent beamwidth, and $\beta$ is the angle between the magnetic axis and the observer's direction. We find that eqs. (2), (4) and (8) form a complete set of geometrical restrictions which the six variables $\alpha, \beta$, $\rho, \Delta \phi, \Delta \psi,(d \psi / d \phi)_{\max }$ must satisfy. In short, parameters $\alpha, \beta$ and $\rho$ are completely determined by $\Delta \psi, \Delta \phi$ and $(d \psi / d \phi)_{\max }$ because of equations (2), (4) and (8). This forms our geometrical determination method of pulsar parameters from one selfconsistent model of the polar cap.

\section{Formal derivation}

Using eqs.(2) and (4), eq.(8) becomes

$$
\tan \Delta \psi=\frac{\left(\frac{d \psi}{d \phi}\right)_{\max } \sin \phi}{1+\left(\frac{d \psi}{d \phi}\right)_{\max }(1-\cos \Delta \phi) \cos (\alpha+\beta)}
$$

which leads naturally to a surprisingly useful result

$$
\cos (\alpha+\beta)=\frac{\frac{(d \psi / d \phi)_{\max } \sin \Delta \phi}{\tan \Delta \psi}-1}{\left(\frac{d \psi}{d \phi}\right)_{\max }(1-\cos \Delta \phi)} .
$$

It is very clear that the variable $\alpha+\beta$ can be immediately calculated from the observed measurements of $(d \psi / d \phi)_{\max }, \Delta \phi$ and $\Delta \psi$. Furthermore, eq.(10) is equivalent to the following two equations

$$
\begin{aligned}
\tan \alpha & =\frac{\left(\frac{d \psi}{d \phi}\right)_{\text {max }} \sin (\alpha+\beta)}{1+\left(\frac{d \psi}{d \phi}\right)_{\text {max }} \cos (\alpha+\beta)} \\
\tan \beta & =\frac{\sin (\alpha+\beta)}{\left(\frac{d \psi}{d \phi}\right)_{\text {max }}+\cos (\alpha+\beta)} .
\end{aligned}
$$

Thus we have arrived at a simple analytic solution of $\alpha, \beta$ and $\rho$ from the observed data through eqs.(10), (11), (12) and (4). The calculations are simpler if we use an expression equivalent to eq.(4)

$$
\sin \rho=\frac{\sin \alpha \sin \Delta \phi}{\sin \Delta \psi} .
$$

Eqs.(10), (11), (12) and (13) show a logical, clear method for determination of the pulsar parameters $\alpha, \beta$ and $\rho$.

\section{Discussion}

Although nearly 500 pulsars have been discovered, polarization measurements are presently available for only about 100 , and not all of these measurements can be used. For the polar cap model, the linear polarization position angle typically follows a simple, frequency-independent, 'S-shaped' trajectory across the pulse profile (Lyne, Smith, and Graham 1971, Manchester 1971). The observed data $(d \psi / d \phi)_{\max }, \Delta \phi$ and $\Delta \psi$ must be consistent with the polar cap model. If $(d \psi / d \phi)_{\max }$ and $\Delta \psi$ are considered to be reliable, there is a strict requirement on the possible range of the observed $\Delta \psi$. A rough estimation (Lyne and Manchester 1988) is

$$
\tan \Delta \psi=\left(\frac{d \psi}{d \phi}\right)_{\max } \sin \Delta \phi,
$$

which can be treated as an approximation of our eq.(9) when $(d \psi / d \phi)_{\max }(1-\cos \Delta \phi)$ is relatively small. A strict analysis involves the use of eq.(9). Using $\Delta \phi$ and $(d \psi / d \phi)_{\max }$ as parameters, the polarization-angle swing $2 \Delta \psi$ is a function of the magnetic inclination, $\alpha$ (figures 1, 2, 3 and 4). So it is evident that there is a possible range of observed parameters. This self-consistency condition can be calculated when eq.(10) is set to 1 . Thus

$$
\left|\frac{\frac{(d \psi / d \phi)_{\max } \sin \Delta \phi}{\tan \Delta \phi}-1}{\left(\frac{d \psi}{d \phi}\right)_{\max }(1-\cos \Delta \phi)}\right| \leq 1
$$

for all the pulsars of our interest. Figures 1 to 4 show that:

1. The curves of $\Delta \psi$ vs. $\alpha$ are generally flat with respect to the variation of $\alpha$ when the values of $\Delta \phi$ are small, even when the values of $(d \psi / d \phi)_{\max }$ are large (cf. figure 1). It is means that we cannot estimate the value of $\alpha+\beta$ from eq.(10);

2. For the median value of $\Delta \phi$, the curves of $\Delta \psi$ vs. $\alpha$ are tlat for small values of $(d \psi / d \phi)_{\max }$, but steep for large values of $(d \psi / d \phi)_{\max }$ (cf. figure 2).

3. For the larger values of $\Delta \phi$ the curves become steeper (cf. figures 3 and 4 ), but for small values of $\alpha$ parts of the curve are rather flat. We define an error function $E$

$$
E=\frac{d \alpha}{2 d \Delta \psi}=\frac{1+\frac{\cos \alpha}{(d \psi / d \phi)_{\max } \cos \beta}}{2 \sin ^{2} \Delta \psi \tan \frac{\Delta \phi}{2} \sin (\alpha+\beta)} .
$$

which best explains the variations in our figures as a combined result of the influences of parameters $2 \Delta \psi$ and $(d \psi / d \phi)_{\max }$ : a larger value of $2 \Delta \phi$ means a greater change of $2 \Delta \psi$, and the higher the value of $2 \Delta \psi$ the curve locates, the larger the range of $2 \Delta \psi$ results. Furthermore, we expect the smallest error coefficient $E$ at the center of each curve when $\alpha \sim 90^{\circ}$. Thus if eq.(16) is treated as an error estimation of our calculation of $\alpha$ with respect to small fluctuations in $2 \Delta \psi$, only those pulsars with large 


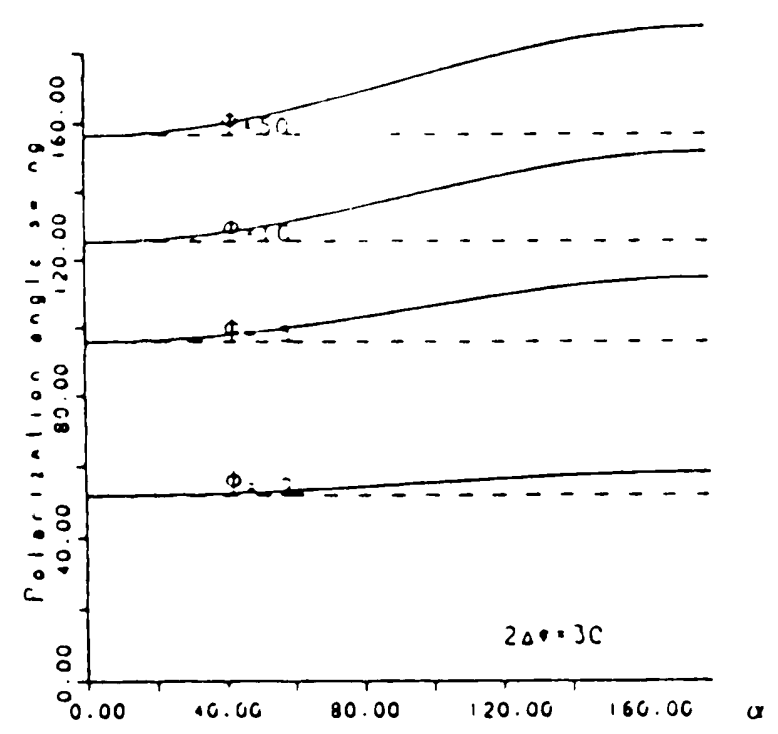

Figure 1 Polarization angle swing $2 \alpha$ as a function of the magnetic inclination $\alpha$ for $\Delta \phi=15^{\circ}$ and selected $(d \psi / d \phi)_{\max }$ values.

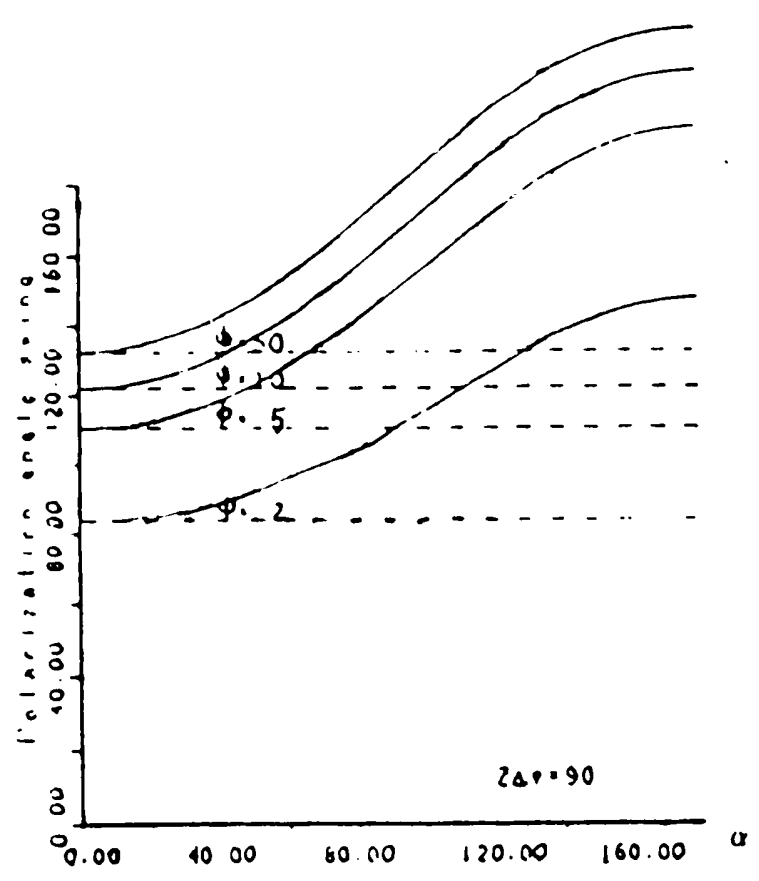

Figure 3 Polarization angle swing $2 \alpha$ as a function of the magnetic inclination $\alpha$ for $\Delta \phi=45^{\circ}$ and selected $(d \psi / d \phi)_{\max }$ values.

pulse width $2 \Delta \phi$, large polarization-angle swing $2 \Delta \psi$ for large central gradient $(d \psi / d \phi)_{\max }$, and large observer angle $\alpha+\beta$ (or large magnetic inclination $\alpha$ ), are of interest for reliable results.

4. We used the pulsar data in Lyne and Manchester's (1988) table 1 and 2. Out of 120 pulsars, 40 satisfy the conditions determined by eq.(15). Of these 40 , there are pulsars with an error coefficient $E$ as large as 50, and 22 pulsars have $E$ values larger

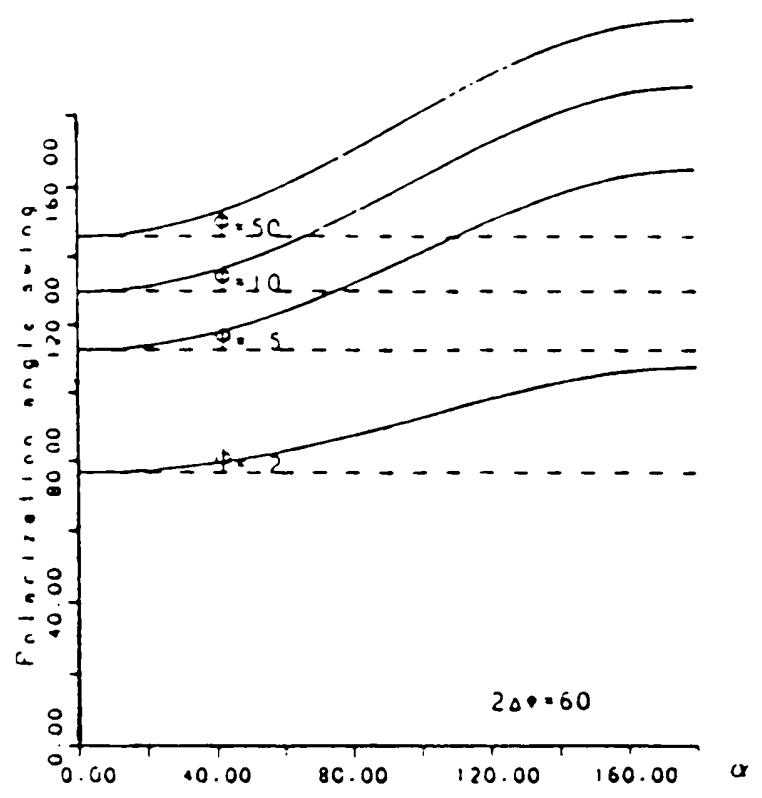

Figure 2 Polarization angle swing $2 \alpha$ as a function of the magnetic inclination $\alpha$ for $\Delta \phi=30^{\circ}$ and selected $(d \psi / d \phi)_{\operatorname{mex}}$ values.

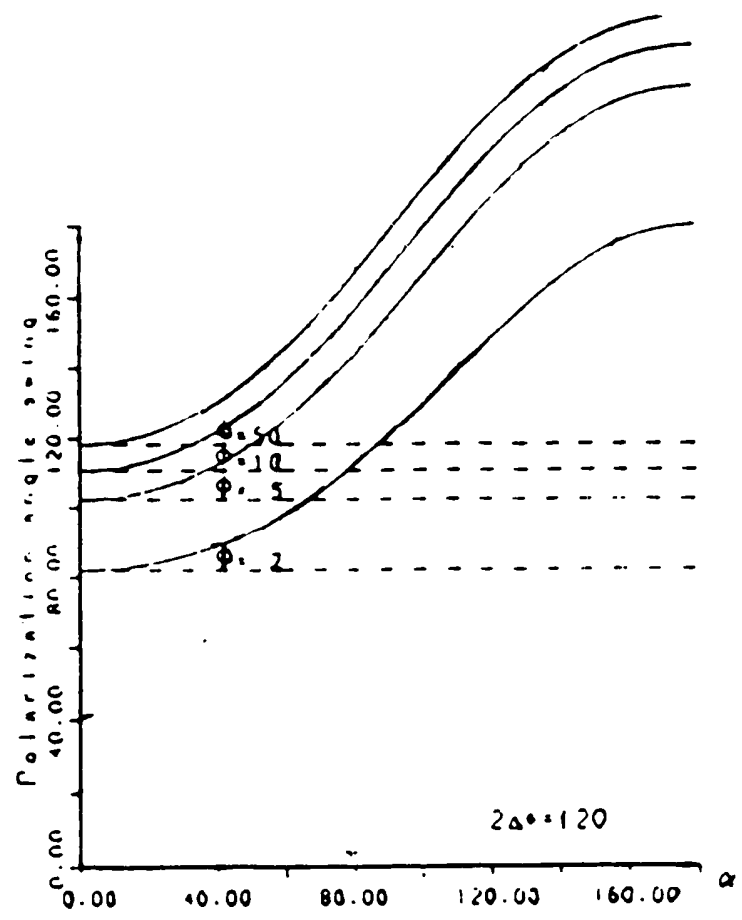

Figure 4 Polarization angle swing $2 \alpha$ as a function of the magnetic inclination $\alpha$ for $\Delta \phi=60^{\circ}$ and selected $(d \psi / d \phi)_{\max }$ values.

than 5. Reliable results were obtained only for those with small error coefficients from eq.(16).

\section{Conclusion}

We have hereby introduced a method to compute pulsar geometry. Our equations are constructed solely on a self-consistent geometrical model of the polar cap. No statistical relations are used. The 
quality of present observations of polarization limits further in the understanding of the observed data. the application of our method. Only a small frac- Our knowledge in this respect is certain to increase tion of pulsars having large magnetic inclination $\alpha$, if better polarization-angle variation measurements large pulse width $2 \Delta \psi$, and large central gradient $\left(\frac{d \psi}{d \phi}\right)_{\max }$ have been amenable to our analysis. However, our method provides a tool which may carry us (especially $\Delta \psi$ ) are obtained.

Acknowledgment: This work was supported by the Natural Science Foundation of China. 